\title{
Maternal effects in gene expression of interspecific coral hybrids
}

\author{
Wing Yan Chan ${ }^{1}$, Jessica Chung ${ }^{2}$, Lesa Peplow ${ }^{1}$, Ary Hoffmann ${ }^{2}$, and Madeleine van \\ Oppen $^{1}$ \\ ${ }^{1}$ Australian Institute of Marine Science \\ ${ }^{2}$ The University of Melbourne Bio21 Molecular Science and Biotechnology Institute
}

May 5, 2020

\begin{abstract}
Maternal effects have been well documented for offspring morphology and life history traits in plants and terrestrial animals, yet little is known about maternal effects in corals. Further, few studies have explored maternal effects in gene expression. In a previous study, F1 interspecific hybrid and purebred larvae of the coral species Acropora tenuis and A. loripes were settled and exposed to ambient or elevated temperature and $p \mathrm{CO}_{2}$ conditions for seven months. At this stage, the hybrid coral recruits from both ocean conditions exhibited strong maternal effects in several fitness traits. We conducted RNA-sequencing on samples from the same experiment and showed that gene expression of the hybrid Acropora also showed clear maternal effects. Only 40 genes were differentially expressed between hybrids and their maternal progenitor. In contrast, 2000 differentially expressed genes were observed between hybrids and their paternal progenitors, and between the reciprocal F1 hybrids. These results indicate that maternal effects in coral gene expression can be long-lasting. Unlike findings from most short-term stress experiments in corals, no genes were differentially expressed in the hybrid nor purebred offspring after seven months of exposure to elevated temperature and $p \mathrm{CO}_{2}$ conditions.
\end{abstract}

\section{Keywords}

Maternal effects, interspecific hybrids, gene expression, RNA-sequencing, coral reefs

\section{Introduction}

Maternal effects can have a large impact on the fitness of offspring. In plants, maternal effects in seed traits (e.g., seed mass, germination time) and offspring fitness (e.g. growth rates) have been well documented (Donohue, 2009). Maternal age at reproduction is known to affect diapause (i.e., suspended development induced by unfavorable environmental conditions) in offspring of insects (Mousseau \& Dingle, 1991), and in amphibians, maternal factors have well known effects in size and rates of development (Warne et al., 2013).

Maternal effects can be the result of maternal provisioning (which is influenced by both environmental and genetic effects), and the direct effects of the environment on epigenetic marks. Maternal provisioning is the supply of nutrients, resources and hormones by the mother during seed or egg development (Videvall et al., 2016). For example, the amount of stored nutrient reserves in seeds can significantly influence early seedling growth and development (Slot et al., 2013). Maternal effects can also manifest via the seed coating (which is maternally produced), the endosperm (which is a triploid tissue with two-third of genotype from the maternal parent), and/or via direct maternal effects on dispersal (Donohue, 2009). For instance, flowering time in Campanula americana determines whether the progeny will germinate in autumn or spring (Galloway \& Etterson, 2007). For many marine larvae, maternal provisioning of lipids is the major source of endogenous energy and this accounts for $\sim 40 \%$ of the metabolic needs of coral larvae (Harii et al., 2010). Maternal provisioning is affected by both the genotype and the environmental conditions experienced by the mother. For example, maternal exposure to hormones can change egg and larval morphology of reef fishes (McCormick, 
1999). Maternal effects due to provisioning generally decrease over time (Roach \& Wulff, 1987), but can also persist through the entire life cycle of an organism. Genomic imprinting is the epigenetic silencing (e.g., via cytosine methylation or chromatin-mediated processes) of one of the parental chromosomes, leaving only expression from the non-silenced chromosome (Alleman \& Doctor, 2000). In the case of maternal effects, only the maternal chromosomes are expressed. Imprinting can be transmitted to one or more subsequent generations (Bischoff \& Müller-Scharer, 2010).

When different genotypes are combined to produce F1 (i.e., first generation) hybrids, maternal effects can affect the phenotypes of $\mathrm{F} 1$ offspring. Hybridization is the crossing between separate species or between strains/lines/populations within a species. The phenotypes of the F1 offspring may be similar to that of their maternal parents (i.e., maternal effects), intermediate between the parents (i.e., additive effects), similar to that of the dominant parent (i.e., dominance), or different to both parents (i.e., over-dominance or underdominance) (Chen, 2013; L. Li et al., 2008; Lippman \& Zamir, 2007). For example, environmental conditions experienced by the mother can influence the expression of genes involved of germination in progeny (Donohue, 2009). However, hybrid gene expression studies often only involve hybrid of one direction (Videvall et al., 2016), and hence are unable to separate between dominance effects and maternal effects.

For corals, maternal effects in morphology (Willis et al., 2006), survival (Chan et al., 2018; Isomura et al., 2013) and thermal tolerance (Dixon et al., 2015) have been reported. Chan et al. (2018) showed that interspecific hybrids of the corals Acropora tenuis andAcropora loripes had similar survival and growth to their maternal purebreds, although they exceeded parental performances in some cases. The bacterial and microalgal endosymbiont (Symbiodiniaceae spp.) communities associated with these corals did not differ between the reciprocal hybrids and their maternal and paternal purebreds (Chan et al., 2019). Since these microorganisms carry vital functions to the coral hosts and can contribute to holobiont fitness differences (Blackall et al., 2015; Rosenberg et al., 2007), this finding suggests that the microbial communities were unlikely responsible for the observed holobiont fitness differences, and that these are likely underpinned by coral host genetic and/or non-genetic transgenerational factors .

The aim of this study was to test if the phenotypic differences in reciprocal F1 hybrids of the corals $A$. tenuis and A. loripes (Chan et al., 2018) could be linked to patterns of gene expression. Four offspring groups (i.e., reciprocal $\mathrm{F} 1$ hybrids and two parental purebreds) were previously produced via a laboratory cross of $A$. tenuis and $A$. loripes and were exposed to seven months of ambient or elevated temperature and $p$ $\mathrm{CO}_{2}$ conditions (Chan et al., 2018). Using samples from the same experiment, we tested for maternal effects in gene expression, as observed in hybrid survival and growth. In addition, gene expression was examined between temperature $/ p \mathrm{CO}_{2}$ conditions within each offspring group.

\section{Materials and methods}

Experimental design and sample collection

Parental coral colonies of $A$. tenuis and A. loripes were collected from Trunk Reef (18deg35'S,

146deg80'E), central Great Barrier Reef in November 2015 and crossed in the laboratory to form two F1 hybrid and two parental purebred offspring groups (see (Chan et al., 2018) for detailed crossing protocol and experimental design). The abbreviation of the offspring groups throughout this study are: TT (purebred A. tenuis ), TL (hybrid), LT (hybrid) and LL (purebred A. loripes ), where the maternal parent is listed prior to the paternal parent in a genetic cross by convention (Miller et al., 2012). For example, "TL" is a hybrid formed by crossing $A$. tenuis eggs with $A$. loripes sperm. Recruits settled onto ceramic plugs were randomly distributed across two treatment conditions $(\mathrm{n}=12$ replicate tanks per treatment, $\mathrm{n}=20$ ceramic plugs per offspring group per tank): ambient conditions (27oC and $415 \mathrm{ppm} p \mathrm{CO}_{2}$ ) and elevated conditions (ambient $+1 \operatorname{deg} \mathrm{C}$ and $685 \mathrm{ppm} p \mathrm{CO}_{2}$ ). Given the predicted sea surface temperature (SST) increase in coral reefs ranges from $\sim 1.4$ and $\sim 3.6 \mathrm{degC}$ by the year 2100 (under RCP 2.6 and 8.5 respectively and relative to pre-Industrial period) (Bindoff et al., 2019), an elevated temperature of $+1 \mathrm{oC}$ to present day ambient temperature reflects a realistic scenario that will likely occur in the coming decades. Note that present day SST has already increase by $\sim 0.9 \mathrm{deg} C$ since pre-industrial time (Bindoff et al., 2019). 
Coral recruits were reared under treatment conditions in filtered seawater for seven months at the National Sea Simulator of the Australian Institute of Marine Science. A microalgal diet supplement was supplied to the corals daily and their fitness traits were measured. To mimic the natural environment as closely as possible, the experimental conditions followed diurnal and annual temperature variations of Davies Reef (18.83deg S, 147.63deg E), which is a reef near the collection sites of the parental colonies. At the end of the seven-month experiment, recruits from three tanks of each treatment were randomly selected for sampling. Due to the small size (and therefore low RNA quantity) of individual recruits, multiple recruits of the same offspring group from the same tank were pooled to form one sample. Each pooled sample contained 30 coral polyps. RNA pooling was considered appropriate as the purpose of this study was to examine populationlevel rather than individual-level differences (Davies et al., 2016; Kendziorski et al., 2003). Three pooled samples per offspring group per treatment were collected, except only one sample was available for purebred A. tenuis (TT) under elevated conditions due to high mortality (Table S1). Samples were snap-frozen in liquid nitrogen and stored at $-80 \operatorname{deg} \mathrm{C}$ until RNA extraction.

RNA extraction

Sample tissues were mechanically disrupted prior to RNA isolation. Approximately 30 acid washed glass beads (Sigma, 710-1180 $\mu \mathrm{m}$ diameter) and $600 \mu \mathrm{L}$ RLT buffer (Qiagen) were added to each sample. The samples were then subjected to $2 \times 40 \mathrm{~s}$ cycles of bead beating at $4 / \mathrm{s}$ in a fast Prep-245G (MP Biomedicals). Total RNA was isolated from the sample homogenate using Qiagen RNeasy mini kit (including the optional DNase treatment). Total RNA was eluted in $40 \mu \mathrm{L}$ of RNase free water and $3 \mu \mathrm{L}$ were visualized on a $1 \%$ agarose, $0.5 \times$ TBE gel for quality check. RNA concentration was measured using the Qubit RNA HS Assay (Thermo Fisher Scientific/Invitrogen), with fluorescence analysis on a NanoDrop 3300 Fluorospectrometer (Thermo Fisher Scientific). Between $500 \mathrm{pg}$ and $100 \mathrm{ng}$ total RNA underwent reverse transcription and cDNA was amplified using NuGen's Ovation V2.0 kit (with one cycle amplification). The amplified cDNA was then purified using magnetic beads (Beckman Coulter Agencourt kit) and $1 \mu \mathrm{L}$ was visualized on a $1 \%$ agarose, $0.5 \times$ TBE gel. Purity of sample cDNA was determined by A260/A280 ratios measured with a NanoDrop 2000 spectrophotometer (Thermo Fisher Scientific). cDNA concentration was measured using the Quant-iT PicoGreen dsDNA Assay (Thermo Fisher Scientific/Invitrogen). Sample cDNA concentrations were normalized and $25 \mu \mathrm{L}$ of $20 \mathrm{ng} / \mu \mathrm{L}$ cDNA were sent to Ramaciotti Centre for Genomics (UNSW, Sydney) for Nextera XT Library Preparation and paired-end sequencing on the Illumina NextSeq platform.

\section{Sequence data processing}

Quality and adapter trimming were carried out on raw reads, discarding reads $<50 \mathrm{bp}$ or with an averaged quality score $<20$ in a sliding window of five bases. Since the coral holobiont is associated with high densities of prokaryotes and algal endosymbionts, reads were filtered with the following steps: First, reads were compared to an rRNA database (Silva132_LSU, Silva132_SSU) and matches (i.e., e-values [?] 10 $0^{-5}$ ) were removed using the programSortMeRNA (Kopylova et al., 2012). Second, reads were compared to the algal endosymbiont genome (genus Cladocopium,symC_scaffold_40.fasta (Shoguchi et al., 2018) and matches were removed using bbduk. The remaining reads were used to create ade novo assembly for the each offspring groups and a combined de novo assembly for all four offspring groups using Trinity (Grabherr et al., 2011). Small transcripts of $<400 \mathrm{bp}$ were removed from the assemblies (Kenkel \& Bay, 2017), and the longest isoform of each trinity transcript was obtained. Mitochondrial genes were identified using BLASTn to A. tenuismitochondrial genome (NC_003522.1.fasta (van Oppen et al., 2002)) and were retained in the analysis. The remaining transcripts were then identified by BLASTx searches against the most complete coral gene model (A. digitifera , GCF_000222465.1_Adig_1.1_protein.faa (Shinzato et al., 2011)) and NCBI's nonredundant (nr) protein database, with a e-value cut off [?] $10^{-5}$.

Gene names and gene ontologies (GO) of the transcripts were assigned using BLASTx search against UniProt Knowledgebase Swiss-Prot database (The UniProt Consortium, 2015). Transcript abundance of the samples was then estimated using RSEM , an alignment-based method (B. Li \& Dewey, 2011). Transcript quantification of the samples was performed by aligning reads using bowtie2 (Langmead \& Salzberg, 2012) and estimating abundance with RSEM (B. Li \& Dewey, 2011). For gene expression comparison between 
hybrids and parental purebreds, we tested estimating transcript abundance using the parental assembly of $A$. loripes, as well as the combined assembly produced using all offspring groups. The two methods revealed very similar results (Figure S1), and the result presented here are based on transcript abundance estimated using the parental assembly of $A$. loripes. Due to the small number of samples available for the parental purebred A. tenuis (Table S1), a de novo assembly was not conducted or tested as a basis for transcript abundance estimate. For gene expression comparison between treatments within an offspring group, the de novo assembly of each offspring group was used to estimate transcript abundance. Treatment comparison was not conducted for $A$. tenuispurebreds due to an insufficient number of samples (Table S1).

Statistical analyses

Separate analyses were conducted to compare gene expression between hybrids and parental purebreds, and ambient versus elevated conditions within an offspring group. Transcript abundance of the samples and the $B L A S T$ results were output to $\mathrm{R}$ for statistical analyses using the package limma (Ritchie et al., 2015). Firstly, only transcripts that were of coral origin were retained, as indicated in the BLAST results. Secondly, duplicate transcripts were removed. Thirdly, transcripts that consistently had zero or very low counts were removed using the edgeR build in function filterByExpr, and scale normalization (TMM) was applied. For Principal Components Analysis (PCA), sample raw counts were transformed into log2-counts per million (log-CPM) to account for library size differences. A total of four samples were identified to have very small library size (three A. tenuis purebreds (two under ambient, one under elevated conditions) and one TL hybrid under elevated conditions), and a relative log expression (RLE) plot showed that normalization of these samples was unsuccessful (Gandolfo \& Speed, 2018) (Figure S2). These samples were excluded from the analyses. A heatmap was then used to visualize the 500 most variable genes across samples, based on a calculated matrix of Euclidean distances from the log-CPM.

To fit linear models for comparisons, count data was transformed to log-CPM using the voom function in the limma package. Since no treatment effect was found on gene expression (see Results section), the comparison of hybrids and purebreds combined samples from both treatments. Comparisons were made between: 1) maternal parent LL and its hybrid LT, 2) paternal parent LL and its hybrid TL, and 3) between the reciprocal hybrids LT and TL. The parental purebred TT (A. tenuis ) was not included due to a small sample size $(\mathrm{n}=1$, Table $\mathrm{S} 1)$. Empirical Bayes moderation was then carried out to obtain more precise pairwise comparisons and p-values were corrected using the Benjamini-Hochberg method (Benjamini \& Hochberg, 1995). A gene was considered differentially expressed when $\mathrm{p}_{\mathrm{adj}}<0.05$ and with $\log$-fold change (LFC) $>0.2$ using the treat function in the limma package. The list of differentially expressed genes (DEGs) was exported for gene ontology (GO) analyses (goseq (Young et al., 2010)) and visualized using volcano plots (Blighe et al., 2018). The volcano plots and GO analyses focused on the comparison of 1) paternal parent LL with its hybrid TL, and 2) between the reciprocal hybrid LT and TL only, as these were the pairs with a high number of differentially expressed genes (DEGs) to explore. The p-values were corrected with the Benjamini-Hochberg method (Benjamini \& Hochberg, 1995), and a GO category was considered overrepresented or underrepresented when $\mathrm{p}_{\mathrm{adj}}<0.05$. The $\mathrm{R}$ scripts and dataset for statistical analyses are available in: https://datadryad.org/stash/share/XKMEOA9LQ4A_lBd1zfF-RNmjuJBZrRo3RU07i3gXx1w.

\section{Results}

On average, $\sim 10$ million raw Illumina Hiseq reads were obtained per sample. After quality trimming and removal of rRNA and algal endosymbiont components, an average of $\sim 5.5$ million paired reads were retained per sample. The transcriptome of purebred A. loripes contained $\sim 291 \mathrm{k}$ transcripts, and $\sim 59 \mathrm{k}$ transcripts were left after only retaining the longest isoforms and removal of small transcripts $<400 \mathrm{bp}$. See Table S2 for details of other transcriptomes used for preliminary analysis and evaluating treatment effect. A total of $\sim 35 \mathrm{k}$ transcripts found match in the NCBI nr databased and were of coral origin. Following the removal of duplicates and transcripts that consistently had zero or very low counts, 8800 transcripts were remained and used for downstream analyses.

Transcriptome-wide gene expression of the hybrids was similar to that of their maternal purebreds, yet 
distinct from their paternal purebreds and the reciprocal hybrids (Figures 1-4). Principal component analyses (PCA) showed similar expression patterns of the hybrid LT with its maternal purebred LL under both ambient and elevated conditions (Figure 1). The only exception was one LL purebred sample which showed separation with the others in principle component two (Figure 1). Gene expression of the reciprocal hybrid TL also clustered with its maternal purebred TT (but note that $\mathrm{n}=1$ for TT), and was separated with hybrid LT and its paternal purebred LL under both treatment conditions (Figure 1). Within an offspring group, gene expression did not differ between ambient and elevated conditions (Figure 1). At a log-fold change cutoff of 0.2 , only 40 differentially expressed genes (DEGs) were identified between maternal purebred LL and its hybrid LT (Figure 2). In contrast, almost 2000 DEGs were identified between paternal purebred LL and its hybrid TL, as well as between the reciprocal hybrids LT and TL (Figure 2). Among these 2000 DEGs, the hybrid LT and its maternal purebred LL shared 1343 genes that were differentially expressed from hybrid TL (Figure 2). Mitochondrial genes ( $\sim 30)$ were included in the analysis, but none were found differentially expressed.

Maternal effects on gene expression were also evident in the heatmap of the 500 most variable genes across samples (Figure 3). The only exception was one purebred LL which clustered away from the other LL samples, and this was the same sample the showed separation in the PCA plot (Figure 1,3). Among the DEGs with the highest log-fold change (i.e., four DEGs for paternal purebred LL compared to its hybrid TL, and seven DEGs for hybrid LT compared to hybrid TL with LFC > 5), three were shared genes between the two pairs of comparison (Figure 4). Unfortunately, most of these DEGs were annotated as uncharacterized proteins and hence their potential functions were unknown (Table S3). For gene ontology (GO) analyses, GO category "cytosol" (GO: 0005829) was underrepresented in both the comparisons between paternal purebred LL with its hybrid TL and between the reciprocal hybrids LT and TL, with 90 and 96 DEGs respectively in this category (Table S4). Note that "cytosol" is a very broad GO category and was comprised of 620 genes in this dataset. In addition, the GO category "membrane" (GO: 0016020) was also underrepresented in the comparison between paternal purebred LL and its hybrid TL (Table S4). This was also a broad GO category with 255 genes in this dataset and 27 of which were DEGs.

\section{Discussion}

Maternal effects in coral fitness are reflected in gene expression patterns

Maternal effects in recruit survival and size previously reported for A. loripes x A. tenuis hybrid corals were consistent with their gene expression patterns. At the time when the corals were sampled for gene expression analyses, hybrid LT and its maternal purebred LL had higher survival compared to hybrid TL and its maternal purebred TT (i.e., 36-49\% versus 7-23\%) under both ambient and elevated conditions (Chan et al., 2018). Although the corals did not differ in size at seven months of age, maternal effects on size were evident by one year of age (Chan et al., 2018). In addition to the above study, maternal effects have been reported in other Indo-PacificAcropora hybrid corals obtained via laboratory crossing. These include effects on: 1) morphology of interspecific hybrids from an A. pulchra x A. millepora cross (Willis et al., 2006), 2) survival of interspecific hybrid larvae from an A. florida xA. intermedia cross (Isomura et al., 2013), and 3) thermal tolerance of intraspecific A. millepora hybrid larvae from a higher and lower latitude population. In contrast, paternal effects were found on morphology of natural interspecific hybrids of A. palmata and A. cervicornis from the Caribbean (Vollmer \& Palumbi, 2002), and additive effects on survival (i.e., hybrid survival was intermediate between the parental offspring) were observed in experimentally produced intraspecific hybrids of $A$. milleporafrom a higher and lower latitude cross (van Oppen et al., 2014).

While a few studies have reported maternal effects on coral fitness and morphology, little is known about maternal effects on gene expression. In addition to the coral host, the host-associated microbiome can also have an impact on host gene expression (Barfield et al., 2018; Helmkampf et al., 2019). In our study, however, the bacterial and microalgal endosymbiont communities of the corals were similar at the time of sampling (Chan et al., 2019). The consistency between host gene expression and phenotypic results thus suggests that maternal host-related factors were likely the drivers behind the observed fitness differences. Almost 2000 
differentially expressed genes (DEGs) were found when comparing hybrid LT and its maternal purebred LL to hybrid TL, and maternal effects were evident in these corals based on PCA, heatmap and volcano plots. While a statistical comparison cannot be made back to the parental purebred TT due to small sample size, gene expression of hybrid TL was similar to the only TT sample tested based on PCA and the heatmap and was indicative of maternal effects.

Only a few studies have reported maternal effects in gene expression. Videvall et al. (Videvall et al., 2016) showed that gene expression patterns were distinct between parental populations of 12-week-old seedling of the perennial herb Arabidopsis lyrata, and expression in intraspecific hybrids was frequently more similar to that of the maternal than paternal population. Only 15 DEGs were found between the hybrid produced in one direction and its maternal population, yet $>8800$ DEGs were found when compared to its paternal population (Videvall et al., 2016). Interestingly, maternal effects were weaker in the hybrid cross of the other direction, with 334 and 661 DEGs observed when compared to its maternal and paternal population respectively (Videvall et al., 2016). Only one previous study has examined maternal effects on coral hybrid gene expression and only coral larvae were studied. Consistent with our findings, Dixon et al. (2015) showed that gene expression of intraspecific A. millepora hybrid larvae was similar to that of their maternal population. Up to 2,000 genes in hybrids were found to follow the expression patterns of the maternal population (Dixon et al., 2015). In both studies however, maternal effects were examined in early life stages only (i.e., 12-week-old seedling and 6-day-old larvae). Our results show that maternal effects can continue to influence gene expression of hybrid corals up to the age of at least seven months, indicating the potential long-term nature of maternal effects.

While differences in gene expression patterns were obvious between reciprocal hybrids as well as between hybrid TL and its paternal purebred, it was unclear what pathways and mechanisms were linked to these differences. Gene ontology (GO) analyses revealed underrepresentation of a very broad GO category, "cytosol", in both pairs of comparison, suggesting that a wide range of genes and pathways were potentially involved in the observed holobiont fitness differences. In contrast, clear pathways involved in maternal effects were observed in the intraspecific A. millepora hybrid larvae (Dixon et al., 2015). Analyses of cellular component categories of tolerance-associated genes (i.e., genes for which expression levels prior to stress predicted the probability of larval survival under stress) showed enrichment of nuclear-encoded mitochondrial membrane components in hybrid coral larvae whose parents come from a warmer latitude (Dixon et al., 2015). The most upregulated GO categories were energy production and conversion, and encompassed mitochondrial proteins, suggesting mitochondrial protein variation in larvae may have contributed to maternal effects on thermal tolerance (Dixon et al., 2015). In our study, however, no mitochondrial-related pathways or genes were differentially expressed. The difference in GO associated patterns between these two studies may due to 1) the parental populations chosen for hybridization, and 2) the symbiotic/aposymbiotic nature of the corals. Parental populations of the same species from different latitudes were selected in Dixon et al. (2015), whereas parental populations of two different species from the same reef were chosen for this study. The differences in parental thermal regimes in Dixon et al. (2015) may lead to clearer maternal effects on thermal stress-related GO categories. Moreover, gene expression responses of aposymbiotic larvae (in Dixon et al., 2015) were likely different from coral recruits (in this study) that are associated with a high density of microalgal endosymbionts. Hence, the contrasting results of the two studies are unsurprising.

Gene expression was unaffected by long-term exposure to elevated temperature and $\mathrm{pCO}_{2}$ conditions

Elevated temperature and $p \mathrm{CO}_{2}$ conditions had a negative impact on survival and size of the corals used in this study (Chan et al., 2018), yet gene expression within an offspring group did not differ between ambient and elevated conditions (Figure 1). Nevertheless, gene expression changes under short-term acute stress are commonly found in coral. This often involves the regulation of genes encoding heat shock proteins, ion transport, apoptosis, immune responses and/or oxidative stress (Barshis et al., 2013; Desalvo et al., 2008; Meyer et al., 2011; Ruiz-Jones \& Palumbi, 2017). The absence of DEGs in corals under ambient versus elevated conditions was unexpected and may due to the relatively mild and long-term nature of the treatments. The elevated conditions of this study (ambient $+1{ }^{\circ} \mathrm{C}, 685 \mathrm{ppm} p \mathrm{CO}_{2}$ ) were relatively minor 
compared to some of the longer-term studies (e.g., ambient +7 and $+12{ }^{\circ} \mathrm{C}$ (Maor-Landaw et al., 2017), 856-3880 ppm $p \mathrm{CO}_{2}$ (Vidal-Dupiol et al., 2013)). In addition, gene expression responses of corals under longterm stress have been shown to differ from those under short-term stress. Despite significant differences in $\mathrm{CO}_{2}$ concentration under control and natural $\mathrm{CO}_{2}$ seep sites (i.e., ${ }^{\sim} 355$ versus $998 \mathrm{ppm}$ ), only 61 DEGs were found in A. millepora from the two sites (Kenkel et al., 2017). Similarly, the expression of calcification-related genes changed significantly in A. millepora subjected to short-term (i.e., 3 days) high $p \mathrm{CO}_{2}$ exposure (Moya et al., 2012, 2015), but far fewer DEGs were found as exposure time increased (Moya et al., 2015; Rocker et al., 2015). Since cellular stress gene expression responses can be transient (Kültz, 2003), certain expression changes may only be detectable during the initial exposure and therefore fewer differentially expressed genes are generally found in long-term studies.

\section{Conclusions and future studies}

This study showed that maternal effects manifested as gene expression differences in interspecific hybrids of the coral A.tenuis and A. loripes. We also showed that maternal effects can persist to at least seven months of age in coral and were likely responsible for the phenotypes of F1 hybrids. However, exposure to elevated temperature and $p \mathrm{CO}_{2}$ conditions did not result in differential coral gene expression. Although the composition of bacterial and microalgal endosymbiont communities of these corals was similar under ambient and elevated conditions and between hybrids and purebreds (Chan et al., 2019), these microbes may have expressed different genes and contributed to holobiont phenotypic differences. Other less studied members of the coral holobiont, such as viruses and fungi (that were not examined), may also have contributed to coral survival and size differences. Further, post-transcriptional and epigenetic regulation (e.g., DNA methylation) may have varied between treatments and hybrid and purebreds and may have resulted in phenotypic differences (Dimond et al., 2017). Future studies will benefit from adopting a multi-omics approach and assessing other members of the coral-associated microbiome to explore other mechanisms that underpin the phenotype of the coral holobiont.

\section{Acknowledgements}

We thank P. Buerger, C. Kenkel and P. Laffy for fruitful discussions, and support from the National Sea Simulator team of AIMS. This research was funded by the Paul G. Allen Philanthropies and the Australian Institute of Marine Science (AIMS). WYC acknowledges the University of Melbourne International Research Scholarship and Fee Remission Scholarship. MvO acknowledges Australian Research Council Laureate Fellowship FL180100036.

\section{References}

Alleman, M., \& Doctor, J. (2000). Genomic imprinting in plants: Observations and evolutionary implications. Plant Molecular Biology , 43 (2), 147-161. https://doi.org/10.1023/A:1006419025155

Barfield, S. J., Aglyamova, G. V., Bay, L. K., \& Matz, M. V. (2018). Contrasting effects of Symbiodinium identity on coral host transcriptional profiles across latitudes. Molecular Ecology ,27 (15), 3103-3115. https://doi.org/10.1111/mec.14774

Barshis, D. J., Ladner, J. T., Oliver, T. A., Seneca, F. O., Traylor-Knowles, N., \& Palumbi, S. R. (2013). Genomic basis for coral resilience to climate change. Proceedings of the National Academy of Sciences , 110 (4), 1387-1392. https://doi.org/10.1073/pnas.1210224110

Benjamini, Y., \& Hochberg, Y. (1995). Controlling the false discovery rate: A practical and powerful approach to multiple testing. Journal of the Royal Statistical Society. Series B (Methodological) , 57 (1), 289-300. https://doi.org/10.2307/2346101

Bindoff, N., Cheung, W., Kairo, J., Arístegui, J., Guinder, V., Hallberg, R., Hilmi, N., Jiao, N., Karim, M., Levin, L., O’Donoghue, S., Cuicapusa Purca, S., Rinkevich, B., Suga, T., Tagliabue, A., \& Williamson, P. (2019). Changing ocean, marine ecosystems, and dependent communities. In IPCC special report on the ocean and cryosphere in a changing climate . [H-O Pörtner, DC Roberts, V Masson-Delmotte, P Zhai, M 
Tignor, E Poloczanska, K Mintenbeck, A Alegría, M Nicolai, A Okem, J Petzold, B Rama, NM Weyer (eds.)]. In press.

Bischoff, A., \& Müller-Scharer, H. (2010). Testing population differentiation in plant species - how important are environmental maternal effects. Oikos , 119 (3), 445-454. https://doi.org/10.1111/j.16000706.2009.17776.x

Blackall, L. L., Wilson, B., \& van Oppen, M. J. H. (2015). Coral-the world's most diverse symbiotic ecosystem. Molecular Ecology ,24 (21), 5330-5347. https://doi.org/10.1111/mec.13400

Blighe, K., Rana, S., \& Lewis, M. (2018). EnhancedVolcano: Publication-ready volcano plots with enhanced colouring and labeling . https://bioconductor.org/packages/devel/bioc/vignettes/EnhancedVolcano/inst/doc/EnhancedVolcano.html

Chan, W. Y., Peplow, L. M., Menendez, P., Hoffmann, A. A., \& Oppen, M. J. H. van. (2019). The roles of age, parentage and environment on bacterial and algal endosymbiont communities in Acropora corals .Molecular Ecology , 28 (16), 3830-3843. https://doi.org/10.1111/mec.15187

Chan, W. Y., Peplow, L. M., Menendez, P., Hoffmann, A. A., \& van Oppen, M. J. H. (2018). Interspecific hybridization may provide novel opportunities for coral reef restoration. Frontiers in Marine Science, 5 . https://doi.org/10.3389/fmars.2018.00160

Chen, Z. J. (2013). Genomic and epigenetic insights into the molecular bases of heterosis. Nature Reviews Genetics , 14 (7), 471-482. https://doi.org/10.1038/nrg3503

Davies, S. W., Marchetti, A., Ries, J. B., \& Castillo, K. D. (2016). Thermal and $p \mathrm{CO}_{2}$ stress elicit divergent transcriptomic responses in a resilient coral. Frontiers in Marine Science, 3 . https://doi.org/10.3389/fmars.2016.00112

Desalvo, M. K., Voolstra, C. R., Sunagawa, S., Schwarz, J. A., Stillman, J. H., Coffroth, M. A., Szmant, A. M., \& Medina, M. (2008). Differential gene expression during thermal stress and bleaching in the Caribbean coral Montastraea faveolata . Molecular Ecology ,17 (17), 3952-3971. https://doi.org/10.1111/j.1365294X.2008.03879.x

Dimond, J. L., Gamblewood, S. K., \& Roberts, S. B. (2017). Genetic and epigenetic insight into morphospecies in a reef coral. Molecular Ecology , 26 (19), 5031-5042. https://doi.org/10.1111/mec.14252

Dixon, G. B., Davies, S. W., Aglyamova, G. V., Meyer, E., Bay, L. K., \& Matz, M. V. (2015). Genomic determinants of coral heat tolerance across latitudes. Science , 348 (6242), 1460-1462. https://doi.org/10.1126/science.1261224

Donohue, K. (2009). Completing the cycle: Maternal effects as the missing link in plant life histories. Philosophical Transactions of the Royal Society B: Biological Sciences , 364 (1520), 1059-1074. https://doi.org/10.1098/rstb.2008.0291

Galloway, L. F., \& Etterson, J. R. (2007). Transgenerational plasticity is adaptive in the wild. Science, 318 (5853), 1134-1136. https://doi.org/10.1126/science.1148766

Gandolfo, L. C., \& Speed, T. P. (2018). Rle plots: Visualizing unwanted variation in high dimensional data. PLOS ONE , 13 (2), e0191629. https://doi.org/10.1371/journal.pone.0191629

Grabherr, M. G., Haas, B. J., Yassour, M., Levin, J. Z., Thompson, D. A., Amit, I., Adiconis, X., Fan, L., Raychowdhury, R., Zeng, Q., Chen, Z., Mauceli, E., Hacohen, N., Gnirke, A., Rhind, N., di Palma, F., Birren, B. W., Nusbaum, C., Lindblad-Toh, K., .. Regev, A. (2011). Trinity: Reconstructing a full-length transcriptome without a genome from RNA-Seq data. Nature Biotechnology , 29 (7), 644-652. https://doi.org/10.1038/nbt.1883

Harii, S., Yamamoto, M., \& Hoegh-Guldberg, O. (2010). The relative contribution of dinoflagellate photosynthesis and stored lipids to the survivorship of symbiotic larvae of the reef-building corals. Marine Biology 
, 157 (6), 1215-1224. https://doi.org/10.1007/s00227-010-1401-0

Helmkampf, M., Bellinger, M. R., Frazier, M., \& Takabayashi, M. (2019). Symbiont type and environmental factors affect transcriptome-wide gene expression in the coral Montipora capitata . Ecology and Evolution, 9 (1), 378-392. https://doi.org/10.1002/ece3.4756

Isomura, N., Iwao, K., \& Fukami, H. (2013). Possible natural hybridization of two morphologically distinct species of Acropora (Cnidaria, Scleractinia) in the Pacific: Fertilization and larval survival rates. PLOS ONE , 8 (2), e56701. https://doi.org/10.1371/journal.pone.0056701

Kendziorski, C. M., Zhang, Y., Lan, H., \& Attie, A. D. (2003). The efficiency of pooling mRNA in microarray experiments.Biostatistics , 4 (3), 465-477. https://doi.org/10.1093/biostatistics/4.3.465

Kenkel, C. D., \& Bay, L. K. (2017). Novel transcriptome resources for three scleractinian coral species from the Indo-Pacific.GigaScience, 6 (9). https://doi.org/10.1093/gigascience/gix074

Kenkel, C. D., Moya, A., Strahl, J., Humphrey, C., \& Bay, L. K. (2017). Functional genomic analysis of corals from natural $\mathrm{CO}_{2}$-seeps reveals core molecular responses involved in acclimatization to ocean acidification. Global Change Biology ,24 (1), 158-171. https://doi.org/10.1111/gcb.13833

Kopylova, E., Noe, L., \& Touzet, H. (2012). SortMeRNA: Fast and accurate filtering of ribosomal RNAs in metatranscriptomic data.Bioinformatics , 28 (24), 3211-3217. https://doi.org/10.1093/bioinformatics/bts611

Kultz, D. (2003). Evolution of the cellular stress proteome: From monophyletic origin to ubiquitous function. Journal of Experimental Biology , 206 (18), 3119. https://doi.org/10.1242/jeb.00549

Langmead, B., \& Salzberg, S. L. (2012). Fast gapped-read alignment with Bowtie 2. Nature Methods , 9 (4), 357-359. https://doi.org/10.1038/nmeth.1923

Li, B., \& Dewey, C. N. (2011). RSEM: accurate transcript quantification from RNA-Seq data with or without a reference genome. BMC Bioinformatics , 12 (1), 323. https://doi.org/10.1186/1471-2105-12-323

Li, L., Lu, K., Chen, Z., Mu, T., Hu, Z., \& Li, X. (2008). Dominance, overdominance and epistasis condition the heterosis in two heterotic rice hybrids. Genetics , 180 (3), 1725-1742. https://doi.org/10.1534/genetics.108.091942

Lippman, Z. B., \& Zamir, D. (2007). Heterosis: Revisiting the magic . https://www-clinicalkey-comau.ezp.lib.unimelb.edu.au/\#!/content/playContent/1-s2.0-S0168952506004094

Maor-Landaw, K., Ben-Asher, H. W., Karako-Lampert, S., Salmon-Divon, M., Prada, F., Caroselli, E., Goffredo, S., Falini, G., Dubinsky, Z., \& Levy, O. (2017). Mediterranean versus Red sea corals facing climate change, a transcriptome analysis. Scientific Reports , 7 , 42405. https://doi.org/10.1038/srep42405

McCormick, M. I. (1999). Experimental test of the effect of maternal hormones on larval quality of a coral reef fish. Oecologia ,118 (4), 412-422. https://doi.org/10.1007/s004420050743

Meyer, E., Aglyamova, G. V., \& Matz, M. V. (2011). Profiling gene expression responses of coral larvae (Acropora millepora) to elevated temperature and settlement inducers using a novel RNA-Seq procedure. Molecular Ecology , 20 (17), 3599-3616. https://doi.org/10.1111/j.1365-294X.2011.05205.x

Miller, M., Zhang, C., \& Chen, Z. J. (2012). Ploidy and hybridity effects on growth vigor and gene expression in Arabidopsis thaliana hybrids and their parents. G3: Genes, Genomes, Genetics , 2 (4), 505-513. https://doi.org/10.1534/g3.112.002162

Mousseau, T. A., \& Dingle, H. (1991). Maternal effects in insect life histories. Annual Review of Entomology , 36 (1), 511-534. https://doi.org/10.1146/annurev.en.36.010191.002455

Moya, A., Huisman, L., Ball, E. E., Hayward, D. C., Grasso, L. C., Chua, C. M., Woo, H. N., Gattuso, J.-P., ForeT, S., \& Miller, D. J. (2012). Whole transcriptome analysis of the coral Acropora milleporareveals 
complex responses to $\mathrm{CO}_{2}$-driven acidification during the initiation of calcification. Molecular Ecology,21 (10), 2440-2454. https://doi.org/10.1111/j.1365-294X.2012.05554.x

Moya, A., Huisman, L., Foret, S., Gattuso, J.-P., Hayward, D. C., Ball, E. E., \& Miller, D. J. (2015). Rapid acclimation of juvenile corals to $\mathrm{CO}_{2}$-mediated acidification by upregulation of heat shock protein and bcl-2 genes. Molecular Ecology , 24 (2), 438-452. https://doi.org/10.1111/mec.13021

Ritchie, M. E., Phipson, B., Wu, D., Hu, Y., Law, C. W., Shi, W., \& Smyth, G. K. (2015). Limma powers differential expression analyses for RNA-sequencing and microarray studies. Nucleic Acids Research, 43 (7), e47-e47. https://doi.org/10.1093/nar/gkv007

Roach, D. A., \& Wulff, R. D. (1987). Maternal effects in plants.Annual Review of Ecology and Systematics , 18 (1), 209-235. https://doi.org/10.1146/annurev.es.18.110187.001233

Rocker, M. M., Noonan, S., Humphrey, C., Moya, A., Willis, B. L., \& Bay, L. K. (2015). Expression of calcification and metabolism-related genes in response to elevated $p \mathrm{CO}_{2}$ and temperature in the reef-building coral Acropora millepora .Marine Genomics , 24 Pt 3 , 313-318. https://doi.org/10.1016/j.margen.2015.08.001

Rosenberg, E., Koren, O., Reshef, L., Efrony, R., \& Zilber-Rosenberg, I. (2007). The role of microorganisms in coral health, disease and evolution. Nature Reviews Microbiology , 5 (5), 355-362. https://doi.org/10.1038/nrmicro1635

Ruiz-Jones, L. J., \& Palumbi, S. R. (2017). Tidal heat pulses on a reef trigger a fine-tuned transcriptional response in corals to maintain homeostasis. Science Advances , 3 (3), e1601298. https://doi.org/10.1126/sciadv.1601298

Shinzato, C., Shoguchi, E., Kawashima, T., Hamada, M., Hisata, K., Tanaka, M., Fujie, M., Fujiwara, M., Koyanagi, R., Ikuta, T., Fujiyama, A., Miller, D. J., \& Satoh, N. (2011). Using the Acropora digitifera genome to understand coral responses to environmental change. Nature , 476 (7360), 320-323. https://doi.org/10.1038/nature10249

Shoguchi, E., Beedessee, G., Tada, I., Hisata, K., Kawashima, T., Takeuchi, T., Arakaki, N., Fujie, M., Koyanagi, R., Roy, M. C., Kawachi, M., Hidaka, M., Satoh, N., \& Shinzato, C. (2018). Two divergentSymbiodinium genomes reveal conservation of a gene cluster for sunscreen biosynthesis and recently lost genes. BMC Genomics ,19 (1), 458. https://doi.org/10.1186/s12864-018-4857-9

Slot, M., Palow, D. T., \& Kitajima, K. (2013). Seed reserve dependency of Leucaena leucocephala seedling growth for nitrogen and phosphorus. Functional Plant Biology , 40 (3), 244-250. https://doi.org/10.1071/FP12255

The UniProt Consortium. (2015). UniProt: A hub for protein information.Nucleic Acids Research , 43 (D1), D204-D212. https://doi.org/10.1093/nar/gku989

van Oppen, M. J. H., Catmull, J., McDonald, B. J., Hislop, N. R., Hagerman, P. J., \& Miller, D. J. (2002). The mitochondrial genome of Acropora tenuis (cnidaria; scleractinia) contains a large group i intron and a candidate control region. Journal of Molecular Evolution , 55 (1), 1-13. https://doi.org/10.1007/s00239001-0075-0

van Oppen, M. J. H., Puill-Stephan, E., Lundgren, P., De'ath, G., \& Bay, L. K. (2014). First-generation fitness consequences of interpopulational hybridisation in a Great Barrier Reef coral and its implications for assisted migration management. Coral Reefs ,33 (3), 607-611. https://doi.org/10.1007/s00338-014-1145-2

Vidal-Dupiol, J., Zoccola, D., Tambutte, E., Grunau, C., Cosseau, C., Smith, K. M., Freitag, M., Dheilly, N. M., Allemand, D., \& Tambutte, S. (2013). Genes related to ion-transport and energy production are upregulated in response to $\mathrm{CO}_{2}$-driven $\mathrm{pH}$ decrease in corals: New insights from transcriptome analysis. PLOS ONE ,8 (3), e58652. https://doi.org/10.1371/journal.pone.0058652 
Videvall, E., Sletvold, N., Hagenblad, J., Agren, J., \& Hansson, B. (2016). Strong maternal effects on gene expression in Arabidopsis lyrata hybrids. Molecular Biology and Evolution , 33 (4), 984-994. https://doi.org/10.1093/molbev/msv342

Vollmer, S. V., \& Palumbi, S. R. (2002). Hybridization and the evolution of reef coral diversity. Science, 296 (5575), 2023-2025. https://doi.org/10.1126/science.1069524

Warne, R. W., Kardon, A., \& Crespi, E. J. (2013). Physiological, behavioral and maternal factors that contribute to size variation in larval amphibian populations. PLOS ONE , 8 (10), e76364. https://doi.org/10.1371/journal.pone.0076364

Willis, B. L., van Oppen, M. J. H., Miller, D. J., Vollmer, S. V., \& Ayre, D. J. (2006). The role of hybridization in the evolution of reef corals. Annual Review of Ecology, Evolution, and Systematics ,37 (1), 489-517. https://doi.org/10.1146/annurev.ecolsys.37.091305.110136

Young, M. D., Wakefield, M. J., Smyth, G. K., \& Oshlack, A. (2010). Gene ontology analysis for RNA-seq: Accounting for selection bias.Genome Biology , 11 (2), R14. https://doi.org/10.1186/gb-2010-11-2-r14

\section{Data Citation}

[dataset]Chan, W. Y., Chung J., Peplow, L., Hoffmann, A. A., \& van Oppen, M. J. H. (2020). Maternal effects in gene expression of interspecific coral hybrids. Dryad. https://datadryad.org/stash/share/XKMEOA9LQ4A_BBd1zfF-RNmjuJBZrRo3RU07i3gXx1w.

\section{Data Accessibility}

Data and R scripts for statistical analyses are available in: https://datadryad.org/stash/share/XKMEOA9LQ4A_lBd1zfF-RNmjuJBZrRo3RU07i3gXx1w.

\section{Author's contribution}

W.Y.C., M.J.H.O., L.P. and A.H. designed the study. W.Y.C. and L.P. conducted the experiment. L.P. carried out the laboratory work. J.C., W.Y.C. and A.H. undertook bioinformatic and statistical analyses. W.Y.C. and M.J.H.O. wrote much of the manuscript and all authors contributed to the final edited version of the manuscript.

\section{Figure legends}

Figure 1. Principal component analyses of the offspring groups using normalized counts (i.e., log-CPM) of the 8,880 genes retained post filtering. The maternal parent is listed prior to the paternal parent for the abbreviation of the offspring groups, where " $\mathrm{T}$ " is A. tenuis and " $\mathrm{L}$ " is A. loripes .

Figure 2. ( $a$ ) The number of up or down regulated genes between the pairs of offspring groups ( $p_{\text {adj }}<$ 0.05 and log-fold change $>0.2)$. $(b)$ Venn diagram showing the number of differentially expressed genes (DEGs) between the pairs of offspring groups. The overlapping space between the circles indicates the number DEGs in both pairs of comparison. The abbreviation of the offspring groups is that the first letter indicates maternal parent and the second letter the paternal parent, where "T" is $A$. tenuisand " $\mathrm{L}$ " is $A$. loripes .

Figure 3. Heatmap of the 500 most variable genes across samples based on a calculated matrix of Euclidean distances from the log-CPM. "T" refers to A. tenuis and "L" refers to A. loripes in the offspring group abbreviation, and the maternal parent is listed prior to the paternal parent.

Figure 4. Volcano plots showing the log-fold change and p-values $\left(\mathrm{p}_{\mathrm{adj}}\right)$ of the 8880 genes retained post filtering. Dotted lines indicate the p-value cut off $(<0.05)$ and $\log$-fold change $($ LFC) cut off $(>5)$. Note that the LFC cut off applied throughout this study was 0.2, and the LFC cut off $(>5)$ applied here was only to visualize the genes that were most highly differentially expressed. Genes that pass both p-value and a LFC cut off of $>5$ are in red. Gene names of shared genes between the two pairs of comparison are 
highlighted in blue. (a ) Paternal purebred LL compared to its hybrid TL; $(b$ ) Hybrid LT compared to hybrid TL.
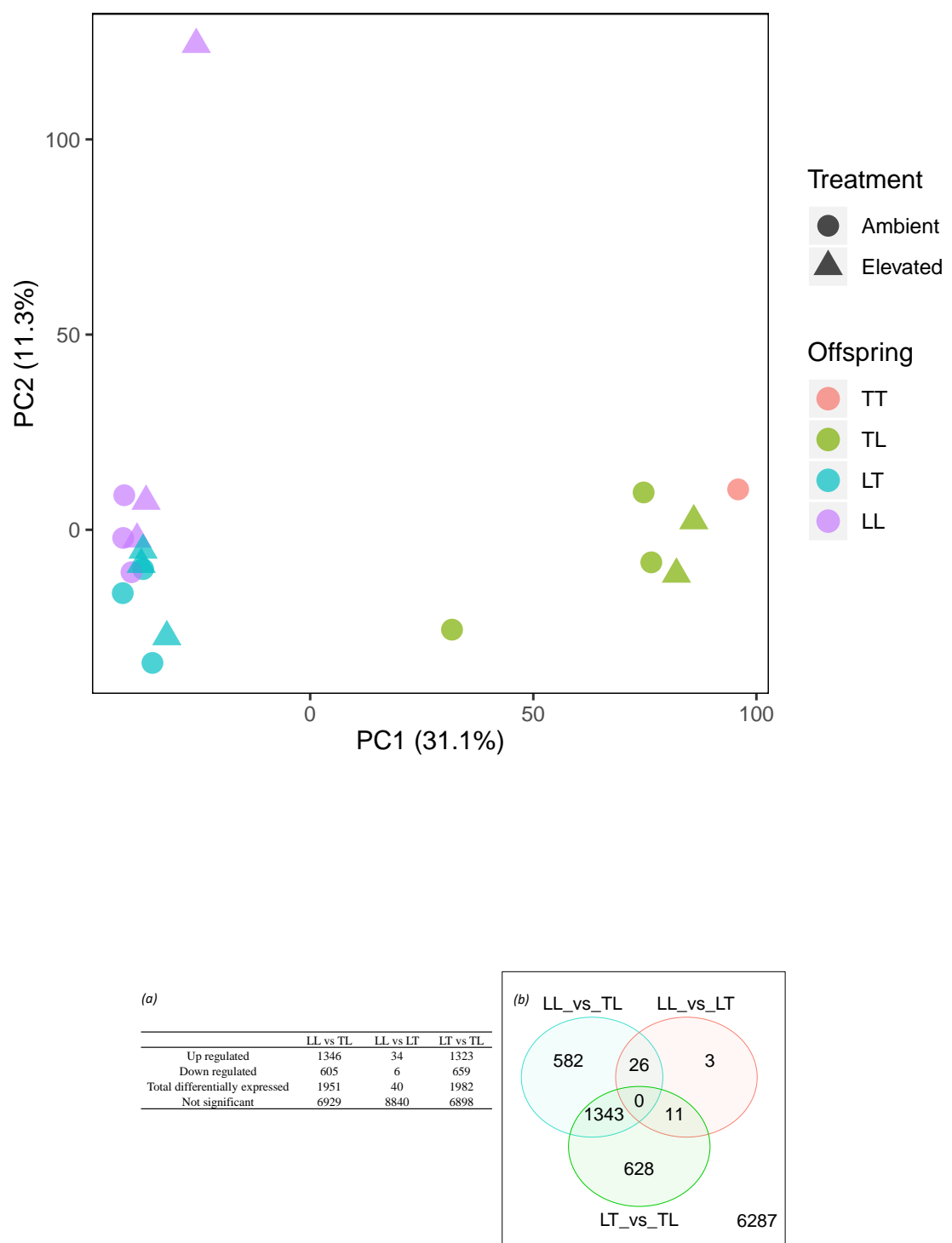


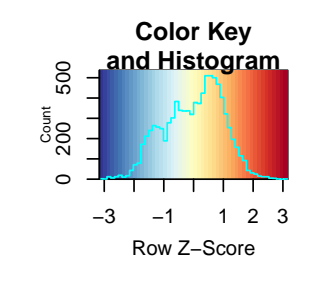

Top 500 most variable genes across samples
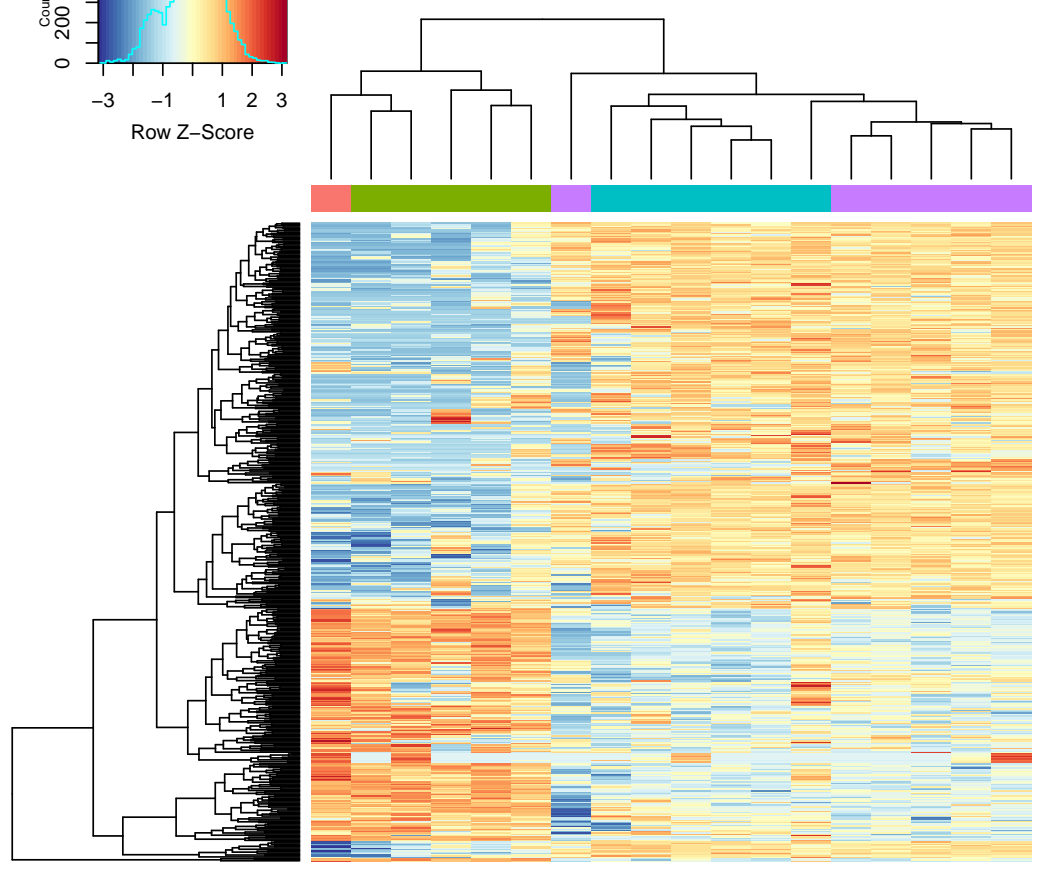

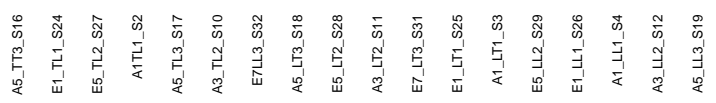

LL vs TL

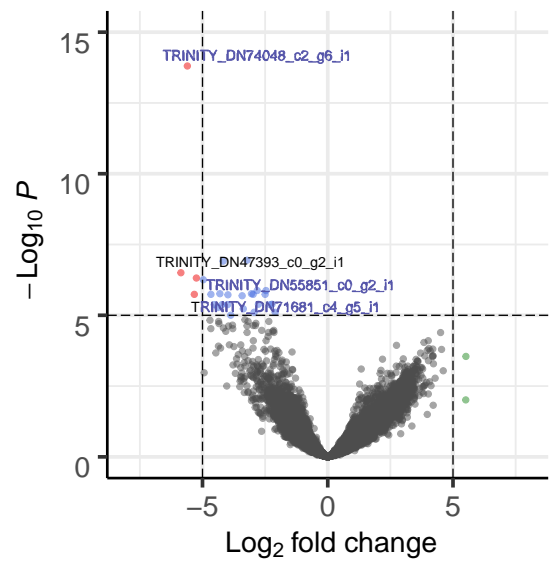

- NS - Log2 FC $\bullet \quad P \quad P \&$ Log2 FC

Total $=8880$ variables
LT vs TL

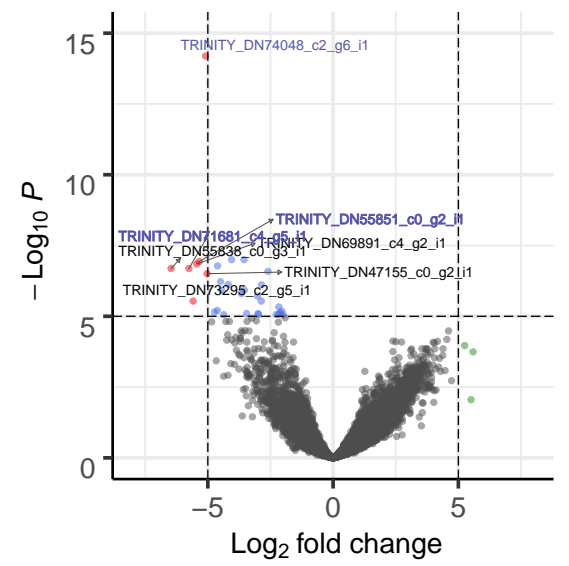

- NS $-\log 2 \mathrm{FC} \bullet \mathrm{P} \bullet \mathrm{P} \&$ Log2 FC

Total $=8880$ variables 